\title{
Humps and superhumps
}

\section{from Andrew King}

CATACLYSMIC variables are close binary systems with orbital periods of a few hours, in which a white dwarf accretes matter from a faint low-mass companion star. They have shown themselves to be ideal systems for studying accretion processes, allowing frequent and stringent tests of theoretical ideas. An important new test is reported by Whitehurst, Bath and Charles on page 768 of this issue.

Cataclysmics show convincing evidence of possessing accretion discs; the accreting matter spirals slowly in to the white dwarf through a sequence of Kepler orbits (centrifugal force balancing gravity) under the action of dissipative forces, gradually giving up its gravitational potential energy to radiation. The stability of such a disc is a matter of great importance and under active debate, with dwarf novae providing the test ground. These are a class of cataclysmics which at irregular intervals (weeks to months) brighten dramatically (factors of about 10 ) in a day or less, remaining at this 'outburst' level for anything up to about 10 days (occasionally longer) before decaying back to their 'quiescent' level in 1 to 3 days. Two types of model have been proposed to explain this behaviour. In one, put forward by G.T. Bath and co-workers (University of Oxford), the 'donor' star (or secondary) is slightly unstable, and at intervals feeds bursts of mass transfer into the accretion disc. In the other, the outburst is a consequence of instabilities purely within the disc, without any change in the state of the secondary: work on these lines is being pursued by groups in Munich, Texas and Santa Cruz (see Nature 302, 12; 1983). In their paper on page 768 , Whitehurst et al. attempt to broaden the debate to include the phenomenon of 'superhumps'.

Superhumps are periodic increases in brightness (of up to 40 per cent) that are observed during the occasional 'superoutbursts', lasting about 12 days, that are additional to the 'normal' outbursts of one subclass of dwarf novae (the SU Ursae Majoris systems). Fascinatingly, the superhumps repeat on a period, $P^{\prime}$, that is a few per cent longer than the orbital period, and so cannot be due to some feature locked to the orbital rotation, such as an eclipse, for example. (It is an intriguing question whether the SU Ursae Majoris phenomenon is restricted to short-period dwarf novae, or whether observational selection effects prevent us recognizing it in longerperiod systems. See Warner, B. in Interacting Binaries, eds Pringle, J.E.P. \& Wade, R., Cambridge University Press; in the press, and van Paradijs, J. Astr. Astrophys. 125, L16; 1983, for a discussion).

Whitehurst et al. focus on two systems which have orbital inclinations high enough that the secondary star periodically crosses our line of sight to the accretion disc, causing a deep eclipse, repeating at the orbital period, $P$. Painstaking efforts by $B$. Warner (Cape Town University) and W. Krzeminski (now Las Campanas) have yielded optical light curves through superoutbursts of these systems. The deep orbital eclipses are still there, indicating that most of the extra superoutburst light comes from the accretion disc. The interesting question which Whitehurst et al. address is whether the superhump light is eclipsed. Since the superhumps 'march' slowly through the orbital light curve on the beat period $\left(1 / P-1 / P^{\prime}\right)^{-1}$ of about $2-3$ days, there are phases when the orbital eclipse falls right on top of the superhump. If the superhump light were totally eclipsed at these phases, the residual light at the bottom of the eclipse should be exactly the same as that at phases where eclipse and superhump do not coincide. If, on the contrary, the superhump light were entirely uneclipsed, this residual light should vary in the same manner as the superhump itself. Using data from several eclipses, Whitehurst et al. conclude that the latter is the case. This virtually forces the site of the superhump phenomenon to be on the secondary star, as only a rather contrived model could restrict it to the small part of the accretion disc which is never eclipsed.

Activity on the secondary star is, of course, exactly what is invoked by the Bath model for dwarf nova outbursts; in the disc instability picture such activity would require a separate explanation. Moreover, most models place the superhump site on the accretion disc, where it would be partially eclipsed (see Warner, op. cit.). Whitehurst et al. suggest that the superhump is due to a bright spot on the secondary where part of the stellar envelope is lost to the white dwarf to cause the superoutburst: the spot is larger than usual since superoutbursts last longer than normal outbursts. The superhump period $P^{\prime}$ then represents the rotation period of the secondary; that is indeed expected to be just slightly longer than the orbital period $P$ because of the synchronizing effect of tidal torques in a close binary.

All this presupposes that one accepts Whitehurst et al.'s conclusion that the superhumps are entirely uneclipsed. Because the superhump does not repeat precisely on each appearance, an escape hatch from this conclusion is still ajar, in that partially eclipsed superhumps may be allowable (see Whitehurst et al.'s Fig. 2). Whether they occur is a question that can be settled observationally, and should soon be, with the powerful stimulus of the new paper. But even when this question is disposed of, others will remain, such as why the superhump radiation pattern has such a highly structured and repeating nature. Superhumps and superoutbursts look set to challenge theoreticians and observers for some time to come.

Andrew King is in the Department of Astronomy, University of Leicester, Leicester LEI $7 R H$.

\section{Molecular biology}

\section{Transposable elements and suppressor genes}

\section{from Ian J. Jackson}

Molecular biologists like mutations. Determination of the molecular basis of genetic mutations has already revealed unsuspected means by which gene expression can be disrupted. Now a class of mutant genes in species as diverse as yeast, Drosophila and mouse is providing more surprises as to mechanisms of mutagenesis, and may well yield information about the regulation of normal development. The mutations are caused by the insertion of transposable genetic elements in or near a gene and are suppressed by recessive genes which map elsewhere in the genome. Recently published work together with data discussed at a meeting in Colorado* reveals a remarkably unified theme among the mutations at the structural level, although the modes of action of the

* UCl.A Symposium, 'Genome rearrangement', held at Steamboal Springs, Colorado, 8-12 April 1984. suppressor genes are proving to be somewhat enigmatic.

The transposable elements that cause the mutations include four classes: Ty of yeast, copia and gypsy of Drosophila and the ecotropic endogenous retroviruses of mouse. Each consists of a few kilobases of DNA flanked at either end by a duplicated sequence of several hundred base pairs, the long terminal repeat (LTR). In all cases where they cause a suppressible mutation, the transposable elements are inserted not into coding DNA but into an intervening or flanking sequence. After insertion, homologous recombination between the two LTRs can result in excision of the internal sequences, leaving a single LTR behind, and often resulting in total or partial reversion of the mutation. In all three species this occurs at a frequency of about $10^{-5}$ $-10^{-6}$ per generation. Suppression of these 